Sayaka Oki, Kagaku-akademî to " yûyô na kagaku ». Fontoneru no yume kara kondoruse no yûtopia he [L'Académie des sciences et « les sciences utiles». Du rêve de Fontenelle à l'utopie de Condorcet]

Nagoya, The University of Nagoya Press, 2011.

\title{
Shizuko Hamada-Poret
}

\section{(2) OpenEdition}

\section{Journals}

Édition électronique

URL : http://journals.openedition.org/dht/1849

DOI : $10.4000 /$ dht. 1849

ISSN : 1775-4194

Éditeur :

Centre d'histoire des techniques et de l'environnement du Cnam (CDHTE-Cnam), Société des élèves du CDHTE-Cnam

\section{Édition imprimée}

Date de publication : 1 décembre 2011

Pagination : 240

ISBN : 978-2-9530779-7-1

ISSN : 0417-8726

\section{Référence électronique}

Shizuko Hamada-Poret, « Sayaka Oki, Kagaku-akademî to "yûyô na kagaku ». Fontoneru no yume kara kondoruse no yûtopia he [L'Académie des sciences et « les sciences utiles ». Du rêve de Fontenelle à I'utopie de Condorcet] », Documents pour l'histoire des techniques [En ligne], 20 | 2e semestre 2011, mis en ligne le 21 septembre 2012, consulté le 23 septembre 2020. URL : http://journals.openedition.org/ dht/1849; DOI : https://doi.org/10.4000/dht.1849

Ce document a été généré automatiquement le 23 septembre 2020

(C) Tous droits réservés 
Sayaka Oki, Kagaku-akademî to " yûyô na kagaku ». Fontoneru no yume kara kondoruse no yûtopia he [L'Académie des sciences et « les sciences utiles ». Du rêve de Fontenelle à l'utopie de Condorcet]

Nagoya, The University of Nagoya Press, 2011.

Shizuko Hamada-Poret

\section{RÉFÉRENCE}

Sayaka Oki, Kagaku-akademî to « yûyô na kagaku ». Fontoneru no yume kara kondoruse no yûtopia he [L'Académie des sciences et « les sciences utiles ». Du rêve de Fontenelle à l'utopie de Condorcet], Nagoya, The University of Nagoya Press, 2011. 
1 Après des études en France menées à l'École des Hautes Études en Sciences Sociales (EHESS), l'auteure, Sayaka Oki est retournée au Japon et a rédigé sa thèse qui fut soutenue à l'Université de Tokyo en $2008^{1}$. Ce livre est fondé sur sa thèse qu'elle a amendée et à laquelle elle a ajouté une huitième partie.

2 Cet ouvrage a pour objectif de reconstituer et analyser l'histoire de l'Académie royale des sciences de Paris, considérée comme la première institution étatique dans le domaine des sciences. Par cette analyse, l'auteure essaie d'élucider comment un groupe de savants est arrivé à obtenir le statut officiel d'institution scientifique moderne. Elle se fonde notamment sur la correspondance de

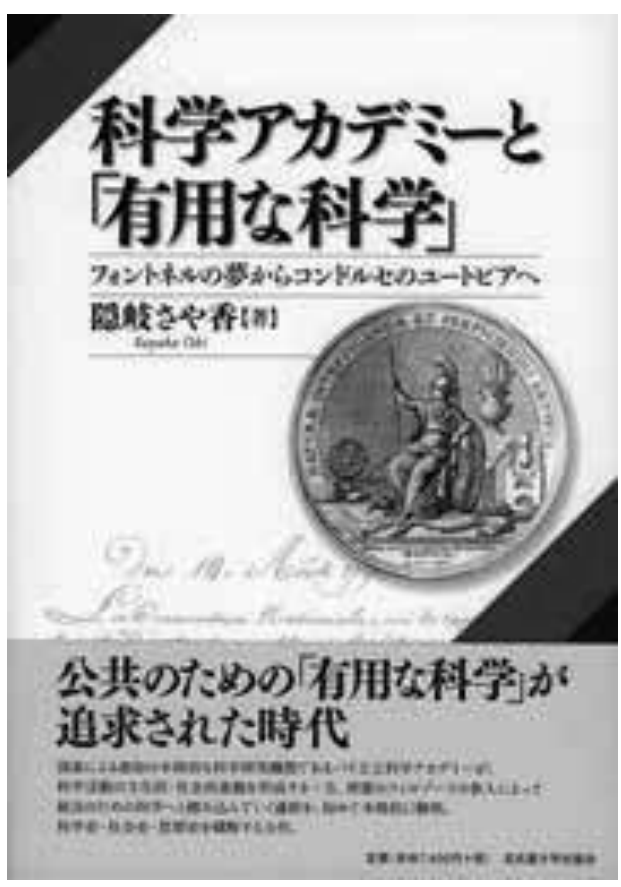
l'institution savante avec l'autorité monarchique.

3 Son travail renouvelle l'historiographie de nouveaux propos et comportements des académiciens que les études précédentes ignoraient, et aident l'auteure à développer ses propres interprétations des faits. Parmi les nouveaux constats de ce livre, l'évaluation des réformes de Turgot est particulièrement intéressante et brillante. Ainsi, au sujet des rôles et du statut des savants de l'Académie dans la relation avec l'autorité administrative, les études précédentes, de Roger Hahn ou de Charles C. Gillispie, ont eu tendance à considérer les réformes de Turgot comme un point de changement décisif. D'après l'auteure, ces réformes ont fissuré la coupole de verre mais ne l'ont pas complètement brisée.

Un autre point de discussion concerne l'expression «sciences utiles » qui donne son titre à l'ouvrage. Rappelant l'emploi fréquent des termes « utilité » et « utile » dans les éloges rédigés par les secrétaires perpétuels de l'Académie comme Fontenelle ou Condorcet, l'auteure entend réinterroger la signification de ce concept pour les académiciens. Selon elle, aux XVIIe et XVIIIe siècles, ni le statut, ni les valeurs des sciences n'avaient de position affirmée comme aujourd'hui. Dans ce contexte, les académiciens réfléchissaient sur ce que doivent être les sciences et leur rôle dans la société. Ainsi, pour justifier la raison d'être de l'Académie, ils invoquaient l' "utilité » des sciences et des arts dans une société publique. Pour comprendre les discours sur l'« utilité», l'auteure fait la distinction entre les différents sens du mot « utilité » pour les académiciens. Elle cite deux sens du mot «utilitarisme»: celui de l'efficacité industrielle, la productivité et la profitabilité commerciale, distinct de celui donné par Jeremy Bentham qui l'emploie dans une acception plus idéologique, connectée à des valeurs sociales et éthiques fortes. La partie consacrée aux différences idéologiques entre d'Alembert et Condorcet d'une part, Buffon d'autre part, éclaire aussi cette distinction.

5 Les éléments mentionnés dans la postface éclairent la démarche de l'auteure. Elle explique qu'elle a commencé une série de recherches sur l'Académie royale des 
sciences après avoir trouvé une catégorie "économie" comme rubrique dans la collection Histoire et Mémoires de l'Académie royale des sciences. Elle s'est ensuite intéressée à l'expression "sciences utiles » fréquemment utilisée par les académiciens, tels Fontenelle et Condorcet. Mais sa motivation pour réaliser cet ouvrage provient aussi de la réflexion sur la réforme de l'enseignement supérieur en cours d'application au Japon, qui bouleverse le milieu scientifique et conduit à interroger la manière dont on conçoit actuellement l'avenir des sciences : pourquoi les hommes poursuivent-ils des recherches en sciences, comment une société considère-t-elle la recherche scientifique, comment place-t-elle sa confiance dans une organisation scientifique et lui donne-t-elle une autorité officielle ? L'intention de l'auteure était donc de trouver, dans l'évolution de l'Académie royale des sciences de Paris, quelques indices pour fournir une réponse à ces questions.

\section{NOTES}

1. Pour ses travaux en français ou en anglais, voir son site internet professionnel (septembre 2011) http://home.hiroshima-u.ac.jp/soki/liste.html ainsi que le site de son tuteur en France, Éric Brian (septembre 2011)

http://eric-brian.blogspot.com/2011/03/72-1024x768-normal-0-21-style.html

\section{AUTEURS}

\section{SHIZUKO HAMADA-PORET}

CDHTE-Cnam 